\title{
Jets from massive protostars: clues on their role in the formation process from masers and high resolution radio / NIR imaging
}

\author{
Francesca Bacciotti ${ }^{* a}$, Fabrizio Massi ${ }^{a}$, Luca Moscadelli $^{a}$, Carmelo Arcidiacono $^{b}$ \\ Alberto Sanna ${ }^{c}$ and Ciriaco Goddi ${ }^{d}$ \\ ${ }^{a}$ Istituto Nazionale di Astrofisica - Osservatorio Astrofisico di Arcetri, \\ Largo E Fermi 5, 50125 Firenze, Italy \\ ${ }^{b}$ Istituto Nazionale di Astrofisica - Osservatorio Astrofisico e scienza dello Spazio di Bologna \\ Via Gobetti 93/3, 40129, Bologna, Italy \\ ${ }^{c}$ MaxPlanck Institut für Radioastronomie, Auf dem Hügel 69, Bonn, Germany \\ ${ }^{d}$ Department of Astrophysics/IMAPP, Radboud University, PO Box 9010, 6500 GL, Nijmegen, \\ The Netherlands \\ E-mail: fran@arcetri.astro.it, fmassi@arcetri.astro.it, \\ moscadarcetri.astro.it, carmelo.arcidiaconodoabo.inaf.it, \\ asanna@mpifr-bonn.mpg.de, C.Goddieastro.ru.nl
}

\begin{abstract}
Disks and jets are believed to deeply influence the early evolution of low-mass stars, but their role in high-mass $\left(M>7 \mathrm{M}_{\odot}\right)$ star formation is still unclear. To investigate this point we conducted on six high mass stars deep high resolution imaging of the $\mathrm{H}_{2} 2.12 \mu \mathrm{m}$ line emission, which is a tracer of jet shocks, with the LUCI NIR cameras mounted at the Large Binocular Telescope (LBT), with follow-ups with Adaptive Optics correction. The targets were selected among a sample already studied with multi-epoch observations of water masers and multi-frequency radio continuum imaging with VLBI /JVLA at sub-arcsecond resolution [5]. In this way we have been able to obtain a full picture of the system on multiple scales from 10 to $10^{5}$ au from the central star. We detect jet-like $\mathrm{H}_{2}$ signatures associated to all the targeted compact radio sources, and the emission is elongated in the same direction than the innermost radio emission [4]. The properties of these features are completely analogous to the ones associated to low mass stars. Our study confirms the presence of collimated jets in the vicinity of newly formed high-mass stars, pointing to a formation mechanism similar in both stellar mass ranges.
\end{abstract}

14th European VLBI Network Symposium \& Users Meeting (EVN 2018)

8-11 October 2018

Granada, Spain

* Speaker. 


\section{Introduction}

The processes leading to the birth of a high-mass $\left(M>7 \mathrm{M}_{\odot}\right)$ star are still largely obscure. Massive star precursors, in fact, are rare and difficult to observe and the role of disks and jets in this mass range is still unclear. A close investigation of disk/jet systems in the high-mass regime can help establishing if high-mass stars emerge from a scaled-up version of the low-mass formation scenario [2]. Radio interferometric observations of water masers from the BeSSeL (Bar and Spiral Structure Legacy) project [7] have proven to be a powerful probe to study jets and winds from high-mass young stellar objects (YSOs) with sub-arcsec resolution. A subsample of the BeSSeL targets was recently investigated at high resolution and sensitivity by complementing multi-epoch VLBI observations of water masers with multi-frequency $(6,13$, and $22 \mathrm{GHz})$ deep imaging of radio continuum emission with the JVLA, within the framework of the program POETS (Protostellar Outflows at the EarliesT Stages) [5], [8], [6]. This analysis shows that the continuum at 13 and 22 $\mathrm{GHz}$ always exhibits a compact or slightly elongated structure, probably associated with a ionized wind close to the star. The associated water masers (studied with milliarcsecond angular resolution and with $1 \mathrm{~km} \mathrm{~s}^{-1}$ velocity accuracy), located within less than 1000 au from the source, suggest in several cases the presence of a collimated outflow, but in other cases their distribution is more complex and difficult to interpret. Crucial information on a possible jet can only be obtained by linking the sub-arcsec structure with the outflowing gas pattern on a scale of $10^{2}-10^{5}$ au from the driving source, a region which is still heavily embedded in dust and gas. To investigate this region, we carried out very deep sub-arcsecond imaging of a sub-sample of six targets in the $\mathrm{H}_{2}$ emission at $2.12 \mu \mathrm{m}$, a tracer of jet shocks, with the LUCI NIR cameras mounted at the Large Binocular Telescope (LBT). Our results have proven to be a crucial test for the existence of collimated outflows from massive protostars and for the origin of the maser spatial distribution [4].

\section{Results}

The six POETS targets observed in the $\mathrm{H}_{2}$ emission (see Table 1) all present (i) strong water masers; (ii) bolometric luminosity typical of a ZAMS type earlier than B3; (iii) compact (size $\leq$ $1^{\prime \prime}$ ); (iv) weak (flux $\leq 50 \mathrm{mJy}$ ) radio continuum emission with a spectral index typical of ionized thermal jets [5]; (v) distance lower than $4 \mathrm{kpc}$.

The sources have been observed using simultaneously LUCI and LUCI2 IR imagers at LBT in binocular mode to increase sensitivity, with seeing-limited angular resolution of about $0 .^{\prime \prime} 8-1^{\prime \prime}$. The $\mathrm{H}_{2}$ line at $2.12 \mu \mathrm{m}$ is excited in shocks when the protostellar jet impinges against gas condensations of moderate density $\left(\mathrm{n}_{\mathrm{H}_{2}} \sim 10^{5} \mathrm{~cm}^{-3}\right)$, and allows to trace the flow at angular scales from $0 . " 5$ to $1^{\prime}$ from the source. As a follow-up, we further imaged two of the targets with LBT/FLAO Adaptive Optics (AO) correction reaching 0."2 angular resolution. We present here preliminar results for three selected cases.

G092.69+3.08 G092.69+3.08 is a $\mathrm{L}_{\mathrm{bol}}=10^{3} \mathrm{~L}_{\odot}$ high mass young star at $\mathrm{d}=1.6 \mathrm{kpc}$. The combination of our observations is shown in Figs.1 and 2. The compact radio emission has a bipolar structure oriented NE-SW (see dashed line). Higher frequency radio emission and maser spots are only seen in the NE blob, which likely traces the protostar [5]. A weak condensation on the top left of Fig. 1 is probably connected to the main source (along the dotted line). A nebulosity in $\mathrm{H}_{2}$ 


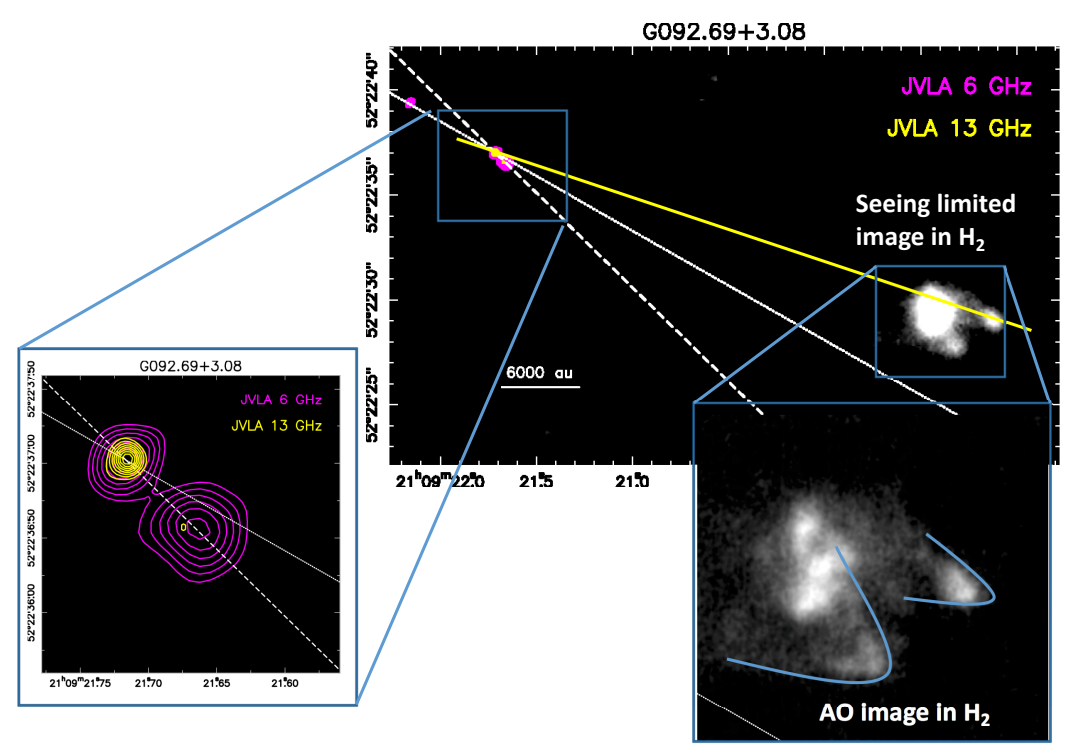

Figure 1: Top-right: LBT/LUCI image in the $\mathrm{H}_{2}$ line at $2.12 \mu \mathrm{m}$ of the G092.69+3.08 region, with superposed contours of the radio emission at $6 \mathrm{GHz}$ (purple) and $13 \mathrm{GHz}$ (yellow) from JVLA observations. The yellow line connects the two main radio blobs with the nebulosity seen in $\mathrm{H}_{2}$. Bottom left: zoom on the two main radio structures. Bottom right: emission in $\mathrm{H}_{2}$ seen with the LBT/FLAO Adaptive Optics correction.

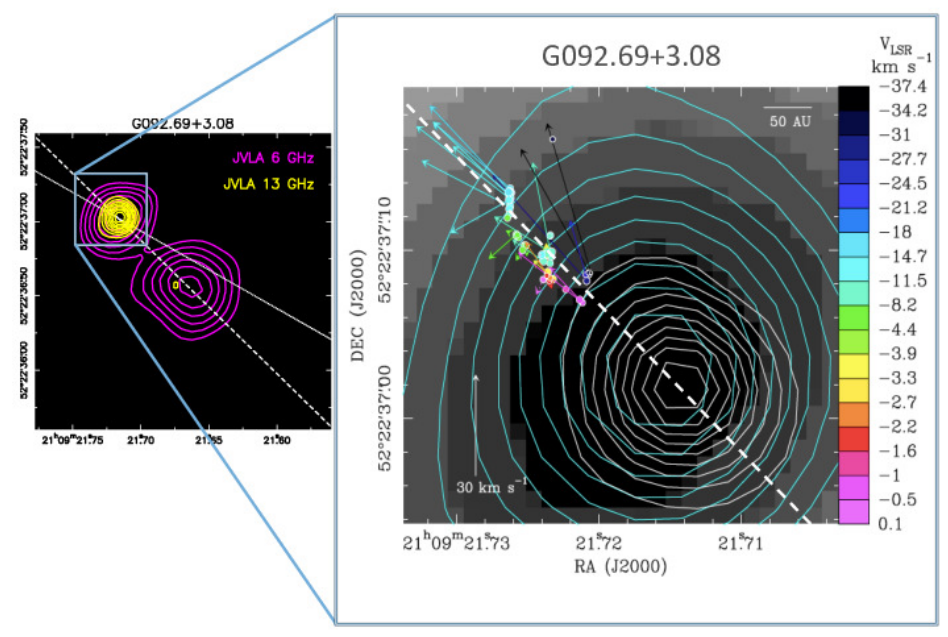

Figure 2: Maser emission in G092.69+3.08, derived from EVN observations (adapted from [5]). The greyscale image in the right panel refers to the the $6 \mathrm{GHz}$ emission, and the cyan and white contours to the $13 \mathrm{GHz}$ and $22 \mathrm{GHz}$ emission, respectively. The masers' radial velocity is color-coded by the scale on the right, and the length and direction of the vectors correspond to the proper motions. The velocity gradient of the maser spots across the system's axis is suggestive of jet rotation [1]. 


\begin{tabular}{|c|c|c|c|c|}
\hline Source name & RA (J2000) & DEC (J2000) & $\mathrm{d}(\mathrm{kpc})$ & $\mathrm{L}_{\text {bol }}\left(\mathrm{L}_{\odot}\right)$ \\
\hline G016.58-0.05 & 18:21:09.1 & $-14: 31: 49$ & $3.6 \pm 0.3$ & $1.310^{4}$ \\
\hline G074 & $20: 25: 07.1$ & $+34: 49: 58$ & $1.59 \pm 0.05$ & $310^{2}$ \\
\hline $\mathrm{G} 075.76+0.34$ & $20: 21: 41.1$ & $+37: 25: 29$ & $3.51 \pm 0.28$ & $1.410^{4}$ \\
\hline G092.69+3.08* & 21:09:21.7 & $+52: 22: 37$ & $1.63 \pm 0.05$ & $110^{3}$ \\
\hline G100.38-3.5 & $22: 16$ & $+52: 2$ & $3.44 \pm$ & $810^{3}$ \\
\hline G111.25-0.77 & $23: 16: 10.4$ & $+59: 55: 29$ & $3.40 \pm 0.18$ & $110^{4}$ \\
\hline
\end{tabular}

Table 1: List of the sources observed in radio continuum, water maser emission and in the $\mathrm{NIR}_{2} \mathrm{H}_{2}$ line filter. The asterisk denotes the targets further observed in the NIR with Adaptive Optics.

emission is located 30 arcsec SW of the radio source, at similar orientation (along the yellow line). The bottom right panel in Fig. 1 shows the emission in $\mathrm{H}_{2}$ imaged with the AO correction. The superposed blue curves guide the eye in the recognition of a double bow-shock structure. Overall, this system appears to host a precessing jet with source coincident with the $13 \mathrm{GHz}$ structure. The clear identification of a jet phenomenon justifies the maser distribution shown in the right panel of Fig. 2. The masers' proper motions are aligned within a small angle toward the NE direction, consistent with shocks along the collimated jet. Even more interestingly, the masers present a gradient in radial velocity across the jet, with more redshifted (blueshifted) emission to the SE (NW) of the jet axis identified by the dashed line. This distribution suggests that the jet rotates around its axis. Evidences of jet rotation have been found recently in several jets from low mass stars, by means of high angular and spectral resolution observations at various wavelengths (e.g. [1], [3]). Jet rotation is a crucial element among the jet properties, as it proves that jets are capable of extracting the excess angular momentum from the forming system, thus allowing the star to accrete up to its final mass. Jet rotation measurements support the widely accepted theories of magneto-centrifugal acceleration and are currently the only mean to determine the jet 'footpoint' area, that is the region in the disk from where the jet originates (see [2] and references therein). For these reasons the observed maser distribution is very interesting and deserves further analysis, which is in progress.

G100.38-3.6. In this source the continuum emission from all bands emerges from the same compact source, to which water masers are associated (Fig. 3). Proper motions cover a variety of angles. The masers might be tracing dense molecular material shocked by the passage of the radioemitting ionized jet. However, this scenario cannot explain the scattered pattern of proper motions nor the positional offset of the radio continuum [5]. We note, however, that the dashed red line follows the orientation of the $\mathrm{H}_{2} 2.12 \mu \mathrm{m}$ line emission at intermediate scales, while the dashed green line indicates the position of distant radio knots located outside the field. The masers' spatial distribution and proper motions appear thus to indicate that the precession of the jet has opened a cavity (with opening angle indicated by the white dashed lines) where jet flow shocks against the cavity walls.

G111.25+0.77 This target is a $\mathrm{L}_{\mathrm{bol}}=10^{4} \mathrm{~L} \odot$ high mass protostar at $\mathrm{d}=3.4 \mathrm{kpc}$. Fig. 4 presents the radio continuum and maser data combined with FLAO-corrected images taken with LBT/LUCI. Jet-like emission with aligned knots seen in the $\mathrm{H}_{2}$ line is clearly associated with the radio con- 


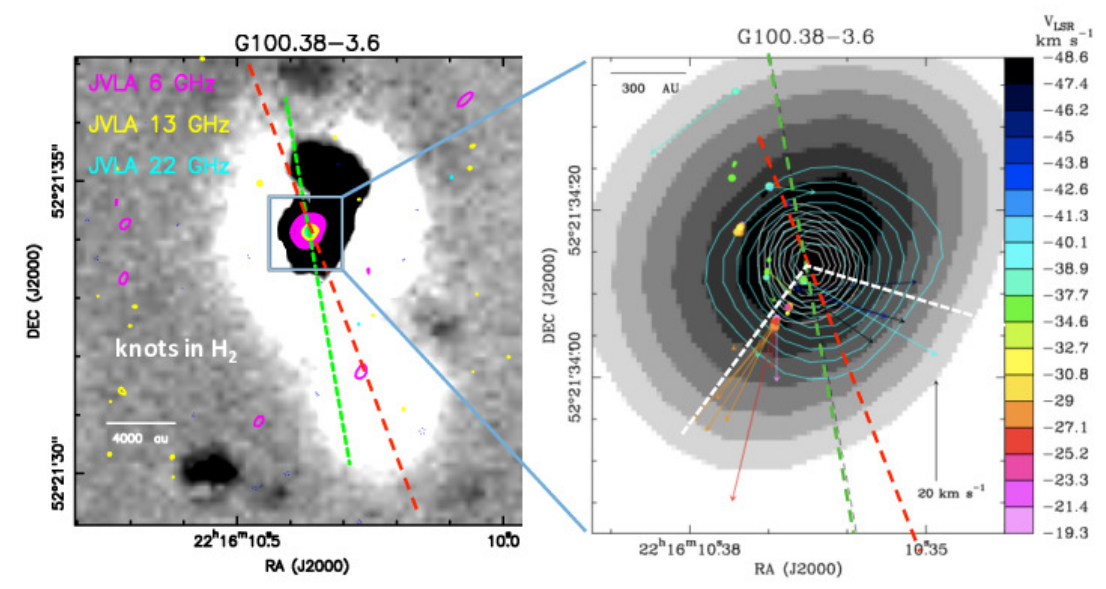

Figure 3: Left: grayscale image of the G100.38-3.6 region taken with LBT/LUCI, showing elongated emission of the $\mathrm{H}_{2} 2.12 \mu \mathrm{m}$ line (white area along the red dashed line). The dashed green line indicates the position of distant knots. Right: zoom of the source region in the radio emission, color-coded as in Fig. 2, adapted from [5]. The cavity opened by the jet is indicated by the white dashed lines.

tinuum compact source and exhibits the same SE-NW orientation. Once again the NIR emission clarifies the nature of the cluster of masers revealed in the right panel [5]. The direction of the masers' proper motions, that point away from the jet axis, can be justified if this well collimated outflow has carved an almost cylindrical cavity through the dense circumstellar material, and the passage of the flow creates shocks against the cavity walls.

\section{Conclusions}

Our AO-assisted LBT/LUCI investigation of the selected POETS high mass targets allowed us to detect $\mathrm{H}_{2}$ line emission at $2.12 \mu \mathrm{m}$, a tracer of jet shocks, associated with all the compact radio sources. The properties of the observed jet-like features are the same than in low mass stars: high collimation, episodic ejections, bow-shocks, precession. The presence of the jet highlighted by the $\mathrm{H}_{2}$ emission can justify some important unexplained properties of the maser distribution, in terms, e.g. of jet rotation imposed by a magneto-centrifugal acceleration mechanism and/or jet-carved cavities in an evolutionary sequence. Overall, our study points to a common formation mechanism for high and low mass stars. 


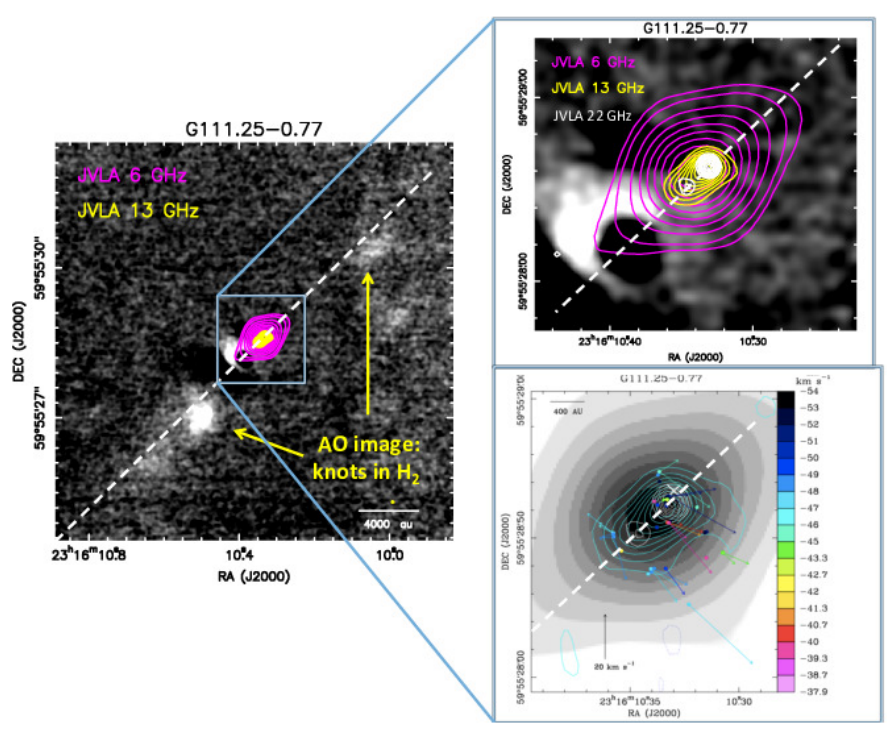

Figure 4: Emission around G111.25-0.77. Left: jet knots in the emission of the $\mathrm{H}_{2}$ at $2.12 \mu \mathrm{m}$ line in the LBT/FLAO-corrected image are found aligned with the radio continuum, zoomed in the top-right panel. Bottom right: $\mathrm{H}_{2} \mathrm{O}$ masers and radio continuum map, coded as in Fig. 2 (adapted from [5]).

\section{Acknowledgments}

The authors acknowledge the LBT-Italian Coordination Facility and funding by the projects PRIN-INAF 2014 Outflows from High-Mass Forming Stars Revealed by Molecular Masers (1.05.01.94.17, P.I. L. Moscadelli) and PRIN-INAF 2016 SKA-GENESIS (1.05.01.88.02, P.I. C. Codella).

\section{References}

[1] Bacciotti, F., Ray, T. P., Mundt, R. et al. HST/STIS Spectroscopy of the Optical Outflow from DG Tauri: Indications for Rotation in the Initial Jet Channel, Astroph J. 576, 222 (2002)

[2] Frank, A., Ray, T. P., Cabrit, S., et al., University of Arizona Press, Tucson, 451, (2014)

[3] Lee, C.-F., Ho, P. T. P., Li, Z.-Y., et al. A rotating protostellar jet launched from the innermost disk of HH 212 Nat. Astr. 1, 152 (2017)

[4] Massi, F., Moscadelli, L., Bacciotti, F., Arcidiacono, C., Sanna, A., Goddi, C., in prep (2019)

[5] Moscadelli, L., Sánchez-Monge, Á., Goddi, C., et al., Outflow structure within 1000 au of high-mass YSOs. I. First results from a combined study of maser and radio continuum emission, Astr. Astroph. 585, 71 (2016)

[6] Moscadelli, L., Sanna, A., Goddi, C. et al. Astr. Astroph., in press, arXiv:1909.08374 (2019)

[7] Reid, M. J., Menten, K. M., Brunthaler, A., et al., Trigonometric Parallaxes of High Mass Star Forming Regions: The Structure and Kinematics of the Milky Way Astroph J. 783, 130 (2014)

[8] Sanna, A., Moscadelli, L., Goddi, et al. Protostellar Outflows at the EarliesT Stages (POETS). I. Radio thermal jets at high resolution nearby H2O maser sources Astr. Astroph. 619, 107 (2018) 